\title{
EMISSION-LINE VARIABILITY IN AGN
}

\author{
DAN MAOZ \\ School of Physics \& Astronomy and the Wise Observatory \\ Tel-Aviv University \\ Tel-Aviv 69978 \\ Israel
}

It has been apparent for some time that comparison of continuum and emission-line light curves of AGN may enable determination of the dimensions and structure of the Broad Line Region (BLR)(see Peterson 1988, and references therein). Observations to this end (Antonucci and Cohen 1983, Peterson et al. 1985, Clavel et al. 1987) and their analysis using cross-correlation to find the time lag between line and continuum variations (Gaskell and Sparke 1986), indicate BLR sizes an order of magnitude smaller than allowed by standard photoionization models for several AGN. Gaskell and Peterson (1987) analyzed errors in the cross-correlation method as applied to AGN time series in general and specifically to the Seyfert galaxy Akn120. In order to generalize and extend their analysis we investigated the significance of BLR sizes derived by cross-correlation under different model assumptions and observational circumstances (Maoz and Netzer 1988).

We first assume a fully known continuum light-curve. Next, a BLR geometry of a given type and size is assumed and an emission-line light curve calculated by accounting for lighttravel-time effects under the simplifying assumption of linear response. To simulate the successful observations of the AGN, the continuum and line light curves are then sampled at random a chosen number of times. A noise of chosen amplitude is added to each "observation" to simulate the effect of measurement errors. The "observed" light curves are interpolated and cross-correlated, the peak of the cross-correlation function giving an estimate of the BLR size based on one experiment. Starting from the random sampling of the synthetic light curves this is repeated many (typically 1000) times in a Monte-Carlo process. By noting the occurences of the cross-correlation peak at all lags computed one obtains an empirical cross-correlation-peak distribution (CCPD). The CCPD shows the probability of obtaining some BLR size from cross-correlation under given conditions.

We examined the effects of a variety of BLR geometries (e.g. spherical vs. flat with different inclinations) on the CCPD. We find that due to the coherent response of nearly face-on flat geometries, their CCPDs are narrower and more highly peaked than those of spherical geometries of equal size. Thus the significance of cross-correlation results is geometry dependent (e.g. a small BLR size deduced from observations may rule out a larger BLR if it is flat and inclined by less than some angle, but not if it is spherical). The CCPD also depends strongly on the thickness of the BLR and its size relative to the assumed continuum variability timescale.

We have applied the method to the best variability data; that of Peterson et al. (1985) for Akn120, and Clavel et al. (1987) for NGC4151. We find that due to the large uncertainties the current observations do not yet conflict with the "standard" photoionization picture. Moreover, we find that reducing the measurement error and greatly increasing the sampling frequency are critical to make future data more significant in sustaining or rejecting current 
models.

We tried to obtain such data in a dedicated period of the Wise Observatory's $1 \mathrm{~m}$ reflector between December 1987 and July 1988 (Maoz et al. 1988). A sample of 14 AGNs was observed spectrophotometrically on an almost nightly basis. The important features of the observations were:

a. A CCD detector with short $(30 \mathrm{~min})$ integrations.

b. Large (20") apertures to avoid systematic effects (Peterson 1988).

c. Low $\mathrm{S} / \mathrm{N}$, low dispersion spectra in the region between $4600-7000 \AA$

d. Simultaneous observation of a nearby (several arcminutes) field star for each object which served as a comparison standard. Thus we achieved photometric accuracy even when observing at high air masses and with adverse weather conditions, unavoidable when attempting to obtain ground-based data with the necessary temporal coverage. The sample included the following "classical" Seyfert 1 galaxies: Akn120, Mrk6, Mrk79, Mrk10, Mrk704, NGC3516, NGC4151, Mrk766, NGC4593, Mrk279, and NGC5548. Most of these previously well-studied AGNs have a known history of variability. In practice, an average spacing of 3-5 days between successful observations, over 5-7 months was obtained for most of the galaxies. Also monitored, though much less intensively, were three QSOs: PG0953+415, 3C273, and PG1634+706.

Preliminary analysis of about two-thirds of the data indicates that until May 1988 none of the above 11 galaxies varied by more than $5-10 \%$ in either the optical continuum or $\mathrm{H} \alpha$ and $\mathrm{H} \beta$. The close spacing of the observations can rule out our having missed any rapid variations. Though this result is discouraging in that no new information concerning BLR structure can be gleaned from it, some tentative conclusions can be drawn:

a. Optical variability in Seyfert $1 \mathrm{~s}$ is less common than usually assumed.

b. Very rapid, high-amplitude variability was not observed, and possibly does not exist, at least not when slower variability is absent.

c. Reliable flux calibration methods, such as that adopted by us, should be used in future studies to increase their accuracy.

\section{REFERENCES}

Antonucci,R.R.J.,\& Cohen,R.D. 1983, Ap.J.,271,564.

Clavel,J.,Altamore,A.,Boksenberg,A.,Bromage,G.E.,Elvius,A.,Pelat,D.,Penston,

M.V.,Perola,G.C.,Snidjers,M.A.J.,\& Ulrich,M.H. 1987, Ap.J.,321,251.

Gaskell,C.M.,\& Peterson,B.M. 1987, Ap.J.Suppl.,65,1.

Gaskell,C.M.,\& Sparke, L.S. 1986, Ap.J.,305,175.

Maoz,D.,\& Netzer,H. 1988, M.N.R.A.S.,in press.

Maoz,D.,Netzer,H.,Brosch,N.,Laor,A.,Leibowitz,E.,Beck,S.,Mendelson,H., Almoznino,E.,\& Mazeh,T. 1988, in preparation.

Peterson,B.M. 1988, P.A.S.P.,100,in press.

Peterson,B.M., Meyers,K.A.,Capriotti,E.R.,Foltz,C.B.,Wilkes,B.J.,\& Miller,H.R. 1985, Ap.J.,292,164. 


\section{DISCUSSION}

WHITTLE If you analyze your data using the techniques developed by Peterson and collaborators in which the [O III] lines are used to normalize the data, do you find apparent variability?

MAOZ We have not yet compared using a star as the reference standard with using the [O III] lines as reference.

ULRICH I would like to make two comments related to the results just presented. First: it is reassuring to see that the emission lines do not vary when the continuum does not vary. Second: the periods of quietness are interesting. The power spectrum of the time variations contain information on the instabilities of the accretion. For this reason, the negative results i.e., observations of non-variability, are important and should be reported. 